\title{
On High Velocity Impact on Carbon Fibre Reinforced Polymers
}

\author{
R. Vignjevic*a, N. Djordjevic ${ }^{\mathrm{a}}$, A. Wasilczuk ${ }^{\mathrm{a}}, \mathrm{T}$. De Vuyst $^{\mathrm{a}}, \mathrm{M} . \mathrm{Meo}^{\mathrm{b}}$ \\ ${ }^{a}$ Brunel University London, Dynamic Response Group, Kingston Lane Uxbridge, UB8 3PH, UK; \\ bUniversity of Bath, Department of Mechanical Engineering, BA2 3LT, UK
}

\begin{abstract}
The gaining popularity of composites and their typical applications (e.g. aerospace, energy and defence) are driving the requirements for the dynamic characterisation of these materials. Carbon fibre reinforced polymers (CFRP), which are the main concern in this work, are composed of stiff, brittle fibres encased in epoxy resin. Their microstructure results in pronounced anisotropy which makes their characterisation challenging even in basic quasi-static mechanical tests. It must be pointed out that the anisotropy and heterogeneity lead to a complexity in behaviour of these materials including a number of failure mechanisms in the material that are activated by different loading conditions. Despite extensive research in the last three decades, a widely accepted and reliable failure theory for composites does not exist [1][2].

The work in progress, presented here, is related to development of the damage part of a constitutive model intended for modelling of high velocity impact on CFRP aerospace structures. The model is based on spectral decomposition of the material stiffness tensor and strain energy. The model development was supported by extensive mesoscale modelling of the effects of physical damage on the damage parameters related to the material deformation eigenmodes. This is done as part of an integrated effort to produce tools for modelling of high velocity impact on composites in the European project EXTREME**.
\end{abstract}

Keywords: High velocity impact, Carbon fibre reinforced polymers, Material model, Hydrocodes

\section{INTRODUCTION}

Here we outline the main features of the damage part of the constitutive model, which is in its final form intended for prediction of dynamic deformation and failure in the composites typically used in aerospace and defence applications. The model, should be able to simulate different architectures of the composite materials. This paper describes the proposed concept applied to unidirectional carbon-fibre reinforced polymer (CFRP) material, which can be conveniently modelled as a transversely isotropic material at the continuum level.

The CFRP materials in the aerospace and defence applications are often exposed to the high rate impact loading, with the strain rates between $10^{3} \mathrm{~s}^{-1}$ and $10^{6} \mathrm{~s}^{-1}$. This is typical for impacts such as: debris impact, hail stone and ice impacts, bird strike, armour penetration etc. These impact cases are characterised by presence of shock waves in the material. In order to design the structures and minimise the risk of their catastrophic failure due to these impacts, it is necessary to understand the material behaviour under such a complex loading and to accurately predict its behaviour.

The damage model was developed in the framework of irreversible thermodynamics with internal variables [3]. The deformation kinematics is limited to small deformations, i.e. relative elongations do not exceed $1 \%$ and rotations do not exceed $0.01 \mathrm{rad}$. This is justified by brittle behaviour of the composites considered. These assumptions make the formulation of the constitutive model relatively simple in terms of the configurational and thermodynamic frameworks. The energy dissipation in the material during deformation process is induced by damage only.

The material is assumed hyperelastic (load path independent behaviour) with transversely isotropic symmetry.

\section{*v.rade@brunel.ac.uk,}

**Acknowledgement: The project leading to this presentation has received funding from the European Union's Horizon 2020 research and innovation programme under grant agreement No 636549. 


\section{DAMAGE MODEL}

A symmetric second order material stiffness tensor can be expressed in general, in terms of six eigenvalues and six corresponding eigentensors (eigenvectors) as [6][7]:

$$
\mathbf{C}=\sum_{\mathrm{i}=1}^{6} \lambda_{\mathrm{i}} \mathbf{v}_{\mathrm{i}} \otimes \mathbf{v}_{\mathrm{i}}
$$

Where $\mathbf{v}_{\mathrm{i}}$ are eigenvectors and $\lambda_{\mathrm{i}}$ is eigenvalues of the tensor defined in 6-dimensional space. These eigenvectors are used to define a set of projection operators $\mathbf{P}_{\mathrm{i}}=\mathbf{v}_{\mathrm{i}} \otimes \mathbf{v}_{\mathrm{i}}$ which are used for the decomposition of the strain tensor $\boldsymbol{\varepsilon}$, into the strain vectors $\boldsymbol{\varepsilon}_{\mathrm{i}}$, which correspond to the material stiffness eigenmodes, i.e. $\boldsymbol{\varepsilon}_{\mathrm{i}}=\mathbf{P}_{\mathrm{i}} \cdot \boldsymbol{\varepsilon}$.

Damage in this constitutive model is represented by a set of scalar variables $d_{i}$ which affect the principal material stiffnesses $\lambda_{\mathrm{i}}$. This is based on the assumption that strain energy corresponding to the individual mode represents free energy for the corresponding damage mode. This definition of damage is consistent with the principle of strain energy equivalence, originally derived by Cordebois and Sidorof [4] as a generalization of pioneering work of Kachanov [5].

Helmholtz free energy of the damaged material defined per unit volume is:

$$
\Psi\left(\boldsymbol{\varepsilon}, \mathrm{d}_{\mathrm{i}}, \mathrm{h}_{\mathrm{i}}\right)=\sum_{\mathrm{i}=1}^{6}\left(\mathrm{~W}_{\mathrm{i}}+\mathrm{H}_{\mathrm{i}}\right)=\sum_{\mathrm{i}=1}^{6}\left[\frac{1}{2}\left(1-\mathrm{d}_{\mathrm{i}}\right)^{2} \lambda_{0 \mathrm{i}} \boldsymbol{\varepsilon}_{\mathrm{i}} \cdot \boldsymbol{\varepsilon}_{\mathrm{i}}+\frac{1}{2} \kappa_{\mathrm{i}} \mathrm{h}_{\mathrm{i}}^{2}\right]
$$

where $\Psi$ is specific free energy, $\boldsymbol{\varepsilon}$ is strain tensor and $h_{i}$ is $i$-th damage mode hardening variable. The energy dissipation rate for each damage mode can be expressed in terms of rate of change of damage related parameters as:

$$
\dot{\Upsilon}_{\mathrm{i}}\left(\varepsilon_{\mathrm{i}}, \mathrm{d}_{\mathrm{i}}, \mathrm{h}_{\mathrm{i}}\right)=-\frac{\partial \Psi_{\mathrm{i}}}{\partial \mathrm{d}_{\mathrm{i}}} \dot{\mathrm{d}}_{\mathrm{i}}-\frac{\partial \Psi_{\mathrm{i}}}{\partial \mathrm{h}_{\mathrm{i}}} \dot{\mathrm{h}}_{\mathrm{i}}=\mathrm{y}_{\mathrm{di}} \dot{\mathrm{d}}_{\mathrm{i}}+\mathrm{y}_{\mathrm{hi}} \dot{\mathrm{h}}_{\mathrm{i}}=\left(1-\mathrm{d}_{\mathrm{i}}\right) \lambda_{0 \mathrm{i}} \boldsymbol{\varepsilon}_{\mathrm{i}} \cdot \boldsymbol{\varepsilon}_{\mathrm{i}} \dot{\mathrm{d}}_{\mathrm{i}}-\kappa_{\mathrm{i}} \mathrm{h}_{\mathrm{i}} \dot{\mathrm{h}}_{\mathrm{i}}
$$

Using maximum energy dissipation principle, it can be shown that the modal damage hardening rate is $\dot{\mathrm{h}}_{\mathrm{i}}=-\dot{\mathrm{d}}_{\mathrm{i}}$. Further using the consistency condition, the damage rate can be defined as:

$\dot{\mathrm{d}}_{\mathrm{i}}=\frac{2\left(1-\mathrm{d}_{\mathrm{i}}\right) \lambda_{0 \mathrm{i}} \boldsymbol{\varepsilon}_{\mathrm{i}} \cdot \dot{\boldsymbol{\varepsilon}}_{\mathrm{i}}}{\kappa_{\mathrm{i}}+\lambda_{0 \mathrm{i}} \boldsymbol{\varepsilon}_{\mathrm{i}} \cdot \boldsymbol{\varepsilon}_{\mathrm{i}}}$

Damage potentials defined for individual modes of deformation are:

$\Phi_{\mathrm{i}}\left(\boldsymbol{\varepsilon}, \mathrm{d}_{\mathrm{i}}, \mathrm{h}_{\mathrm{i}}\right)=\left(1-\mathrm{d}_{\mathrm{i}}\right) \lambda_{0 \mathrm{i}} \boldsymbol{\varepsilon}_{\mathrm{i}} \cdot \boldsymbol{\varepsilon}_{\mathrm{i}}+\left(\kappa_{\mathrm{i}} \mathrm{d}_{\mathrm{i}}-\omega_{0 \mathrm{i}}\right) \leq 0$

Where $\omega_{0 \mathrm{i}}$ is the $i$-th mode initial threshold energy associated with material damage initiation for $i$-th deformation mode, which is determined experimentally by mechanical testing that activates only a single deformation mode. The deformation modes typical for materials with transversely isotropic type of symmetry are shown in Figure 1.

In the current model development, we consider two versions of damage model: one with no damage interaction between the damage modes, and the second version with possible interaction between the modes. The damage related to the principle material stiffnesses controls the evolution of a damage initiation threshold. Evolution of the damage initiation threshold parameters are defined as the separate constitutive laws. For the results presented here a simple set of linear evolution laws was used.

One of the key assumptions, used in the development of this model, is that the material stiffness tensor eigenvectors have constant directions, i.e. are not affected by damage. This is validated using a range of mesoscale models, where different levels of physically representative damage were introduced and its impact on the material stiffness tensor assessed. 


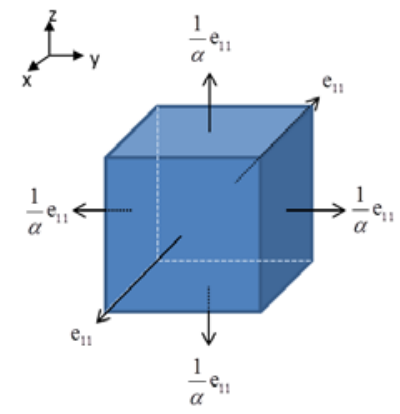

Mode 1

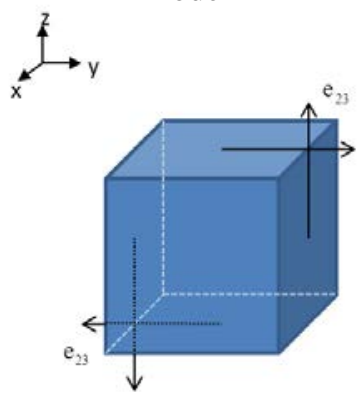

Mode 4

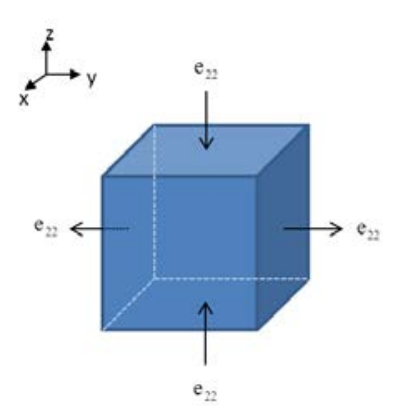

Mode 2

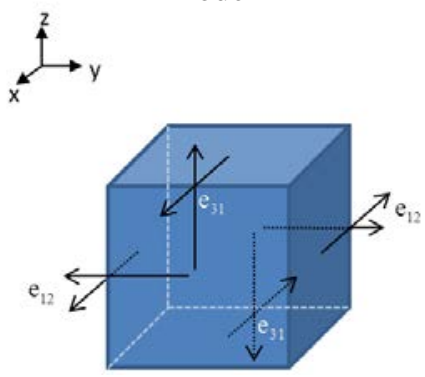

Modes 5/6

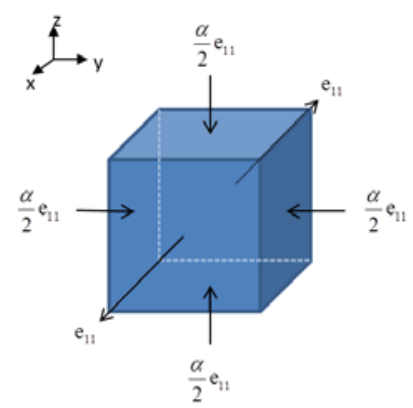

Mode 3
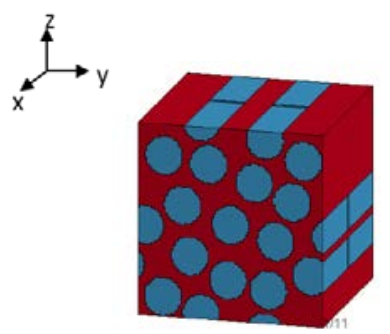

Mesoscale model

Figure 1 Modes of deformation corresponding to eigenvalues of the material stiffness tensor for transversely isotropic material; uniaxial composite with fibres in $\mathrm{x}$ direction and $\alpha \approx 22$ which is representative of CFRP material used in aerospace applications

The effects of fibre damage are illustrated in Figure 2 showing that eigenvectors $\mathrm{v}_{1}$ and $\mathrm{v}_{3}$ rotate by five degrees (limit for small strain approximation) for the value of damage variable $d_{1}=0.4$ which corresponds to fibre failure. In the case of fibre matrix debonding shown in Figure 3 rotation of the eigenvectors does not exceed four degrees and for the case of uniform matrix damage (Figure 4) the rotations of the eigenvectors stay below three and a half degrees. Similar results were obtained for the models with different combinations of these three types of physical damage.
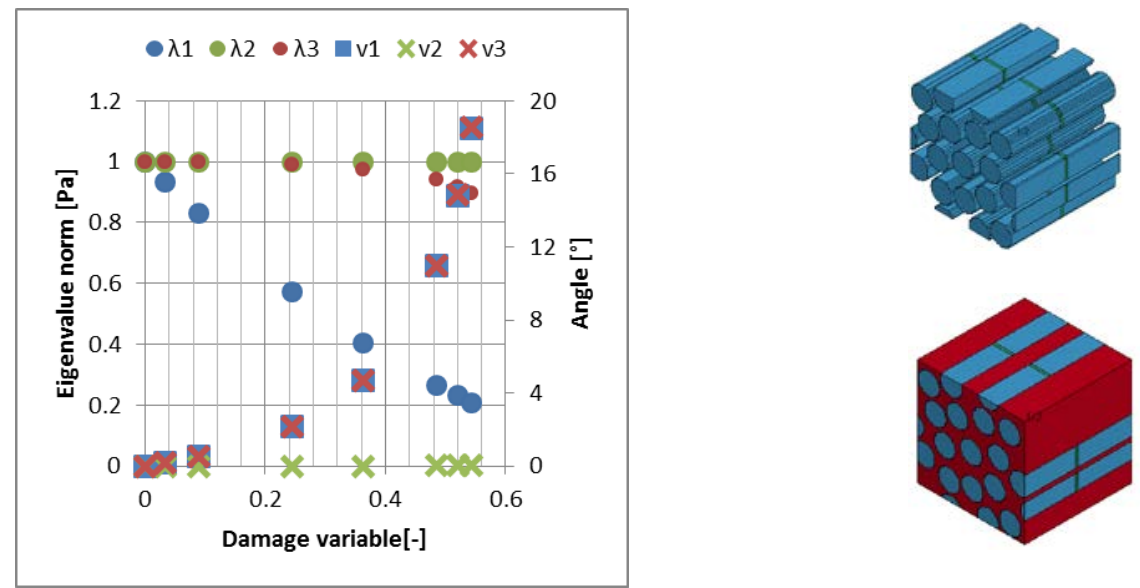

Figure 2 Variation of eigenvalue $\lambda_{1}, \lambda_{2}$ and $\lambda_{3}$ and the rotation of the corresponding eigenvectors v1, v2 and v3 due to fibre failure identified as the main damage mechanism for mode 1 (related to damage parameter $\mathrm{d}_{1}$ ) 


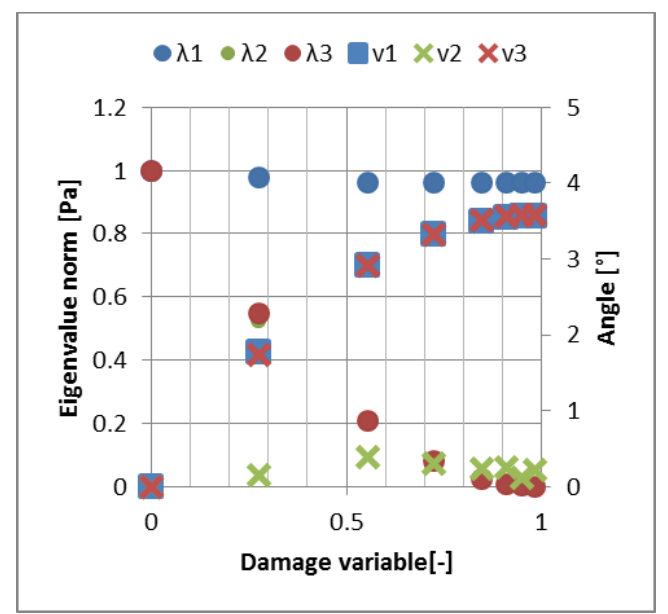

D

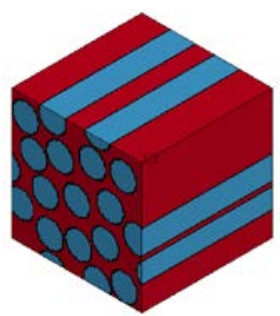

Figure 3 Variation of eigenvalue $\lambda_{1}, \lambda_{2}$ and $\lambda_{3}$ and the rotation of the corresponding eigenvectors v1, v2 and v3 due to fibre matrix debonding identified as a possible damage mechanism for modes $2 \& 3$ (related to damage parameter $\mathrm{d}_{2} \& \mathrm{~d}_{3}$ )
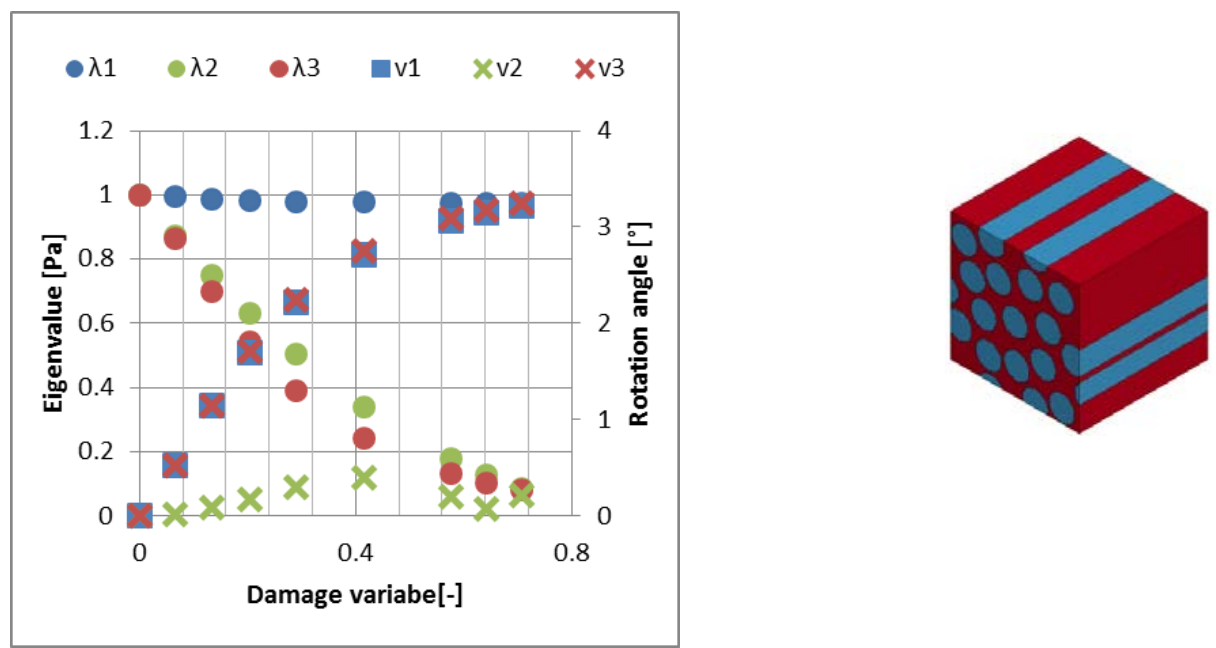

Figure 4 Variation of eigenvalue $\lambda_{1}, \lambda_{2}$ and $\lambda_{3}$ and the rotation of the corresponding eigenvectors v1, v2 and v3 due to uniform matrix damage identified as a possible damage mechanism for modes $2 \& 3$ (related to damage parameter $\mathrm{d}_{2} \& \mathrm{~d}_{3}$ )

\section{INITIAL MODEL VALIDATION}

The first version of the proposed model, which does not allow for damage mode interactions is undergoing validation. The validation process is being performed in three stages. The first stage involves concept validation which includes load cases designed to trigger individual damage modes are considered. This is followed by validation of the model for the load cases designed to trigger mixed damage modes. The final validation stage will involve testing against experimental date from high velocity impact tests. Here we present selection of results from the first stage of testing illustrating that the model performed as conceptually intended.

Damage mode 1 defined by potential $\Phi_{1}=\left(1-\mathrm{d}_{1}\right) \lambda_{1} \overline{\boldsymbol{\varepsilon}}_{1}^{2}+\left(\kappa_{1} \mathrm{~h}_{1}-\omega_{01}\right) \leq 0$ and for $\kappa_{1}=0, \omega_{01}=4.7 \cdot 10^{7}$ and $\lambda_{1}=1.4 \cdot 10^{11}$ models behaviour related to fibre damage and failure as illustrated in Figure 5 . It is important to recall that the material failure coincides with the value $\mathrm{d}_{1}=0.4$ (this limit is introduced to keep damage induced rotation of the eigenvectors below five degrees). 

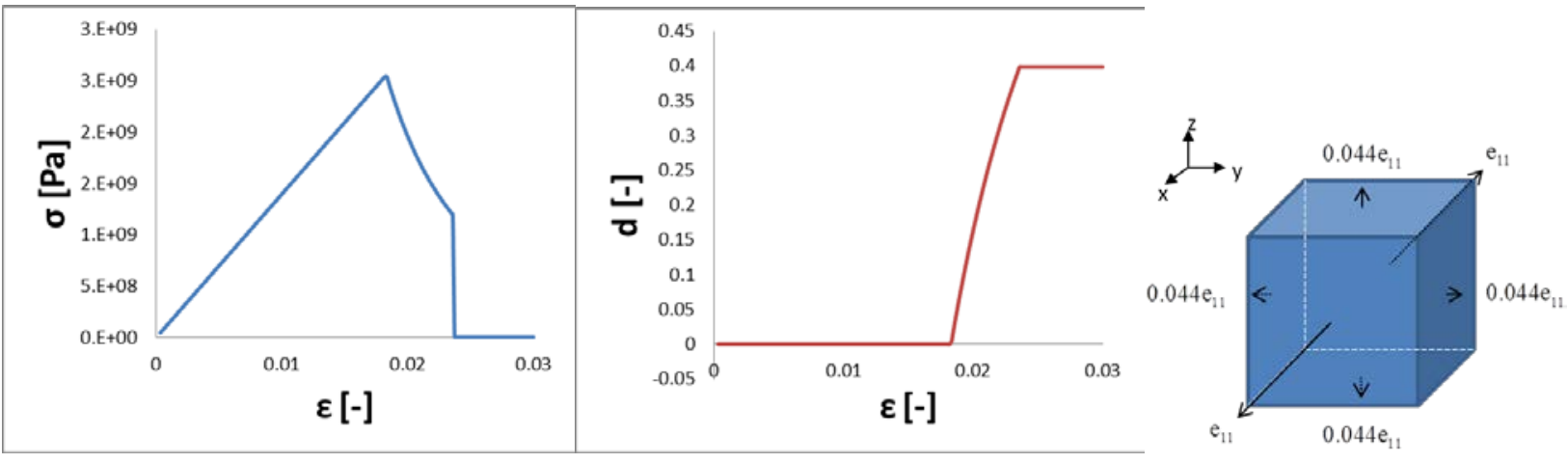

Figure 5 Stress vs strain and damage vs strain curves for the loading condition that corresponds to mode 1

Damage mode 2 is defined by potential $\Phi_{2}=\left(1-\mathrm{d}_{2}\right) \lambda_{2} \overline{\boldsymbol{\varepsilon}}_{2}^{2}+\left(\kappa_{2} \mathrm{~h}_{2}-\omega_{02}\right) \leq 0$ which for $\kappa_{2}=10^{6}$, $\omega_{01}=2.3 \times 10^{5}$ and $\lambda_{1}=5.8 \times 10^{9}$ models behaviour related to the first type of matrix damage and failure as illustrated in Figure 6.
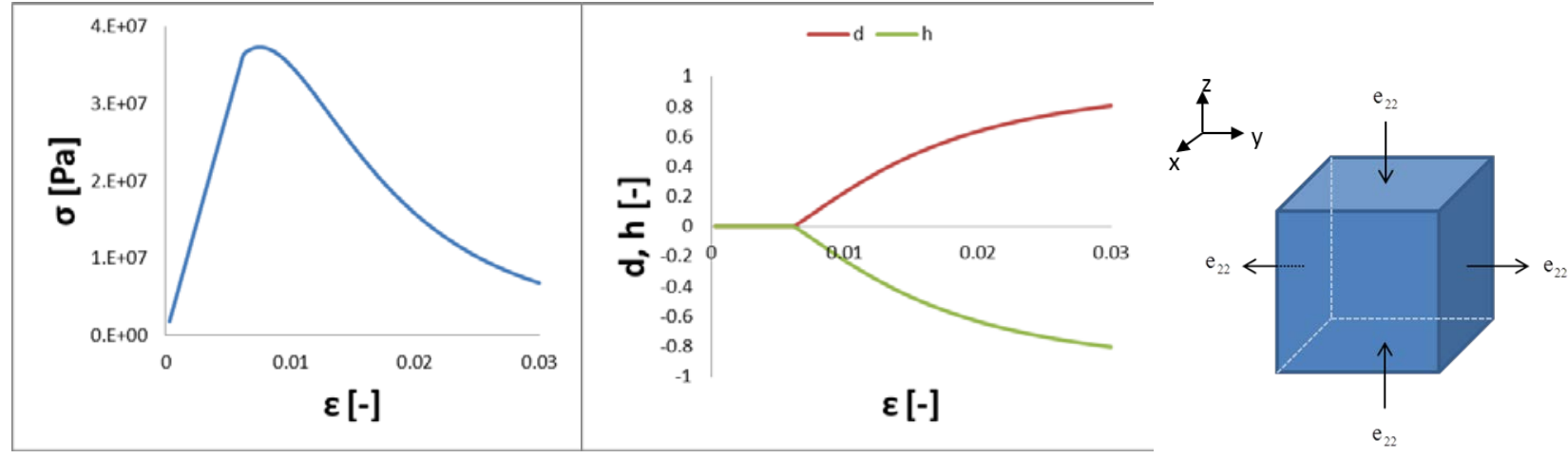

Figure 6 Stress vs strain and damage vs strain curves for the loading condition that corresponds to mode 2

Damage mode 3 is defined by potential $\Phi_{3}=\left(1-\mathrm{d}_{3}\right) \lambda_{3} \bar{\varepsilon}_{3}^{2}+\left(\kappa_{3} \mathrm{~h}_{3}-\omega_{03}\right) \leq 0$ which for $\kappa_{3}=5 \times 10^{7}$, $\omega_{01}=7.9 \times 10^{6}$ and $\lambda_{1}=1.6 \times 10^{10}$ models behaviour related to the second type of matrix damage and failure as illustrated in Figure 7.
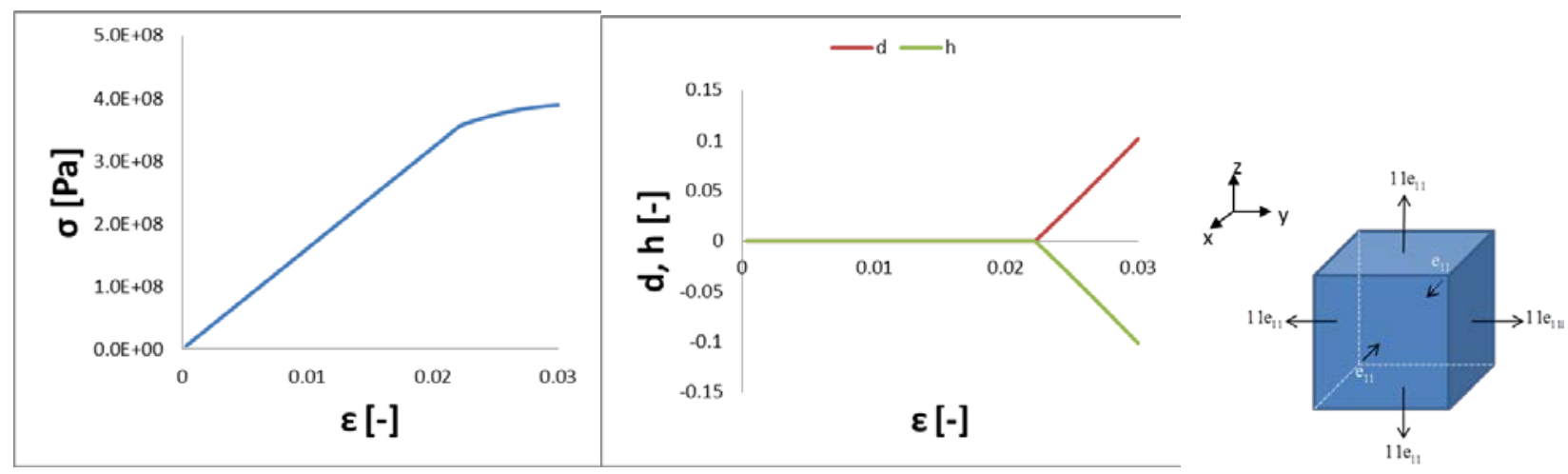

Figure 7 Stress vs strain and damage vs strain curves for the loading condition that corresponds to mode 3 


\section{SUMMARY}

This paper describes work in progress related to development of a material model intended for modelling of high velocity impact on CFRP structures. The model is based on spectral decomposition of the material stiffness tensor and strain energy density. A selection of the validation results of the damage model demonstrate that the model can successfully reproduce damage initiation and evolution related to the loading conditions, which correspond to eigenmodes of the material stiffness tensor for transversely isotropic material.

\section{REFERENCES}

[1] Soden, P. D., Kaddour, A. S. and Hinton, M. J. (2004) 'Recommendations for designers and researchers resulting from the world-wide failure exercise', Composites Science and Technology. Elsevier, 64(3), pp. 589604.

[2] Jones, R. M. (1998) Mechanics of composite materials. CRC press.

[3] Hansen, N. R. and Schreyer, H. L. (1994) 'A thermodynamically consistent framework for theories of elastoplasticity coupled with damage’, International Journal of Solids and Structures. Elsevier, 31(3), pp. 359389.

[4] Cordebois J. P., Sidoroff F. Anisotropic Damage in Elasticity and Plasticity. Journal de mecanique theorique et appliquee, , pp. 45-59 (1980).

[5] Kachanov L. M. Time of the rupture process under creep conditions. Ivz Akad Nauk SSR Otd Tech Nauk, 8, pp. 26-31 (1958).

[6] Schreyer, H. L. (1995) 'Continuum Damage Based on Elastic Projection Operators', International journal of damage mechanics, 4(2), pp. 171-195.

[7] Schreyer, H. L. and Zuo, Q. H. (1995) 'Anisotropic yield surfaces based on elastic projection operators', Trans. ASME: J. Appl. Mech., 62(September), pp. 780-785. doi: 10.1115/1.2897014. 